\title{
Improving Results on Solvability of a Class of $n$ th-Order Linear Boundary Value Problems
}

\author{
Pedro Almenar ${ }^{1}$ and Lucas Jódar ${ }^{2}$ \\ ${ }^{1}$ Vodafone Spain, Avenida América 115, 28042 Madrid, Spain \\ ${ }^{2}$ Instituto Universitario de Matemática Multidisciplinar, Universitat Politècnica de València, Camino de Vera $s / n$, \\ 46022 Valencia, Spain
}

Correspondence should be addressed to Lucas Jódar; ljodar@imm.upv.es

Received 11 April 2016; Revised 9 June 2016; Accepted 19 June 2016

Academic Editor: Elena Braverman

Copyright (C) 2016 P. Almenar and L. Jódar. This is an open access article distributed under the Creative Commons Attribution License, which permits unrestricted use, distribution, and reproduction in any medium, provided the original work is properly cited.

This paper presents a modification of a recursive method described in a previous paper of the authors, which yields necessary and sufficient conditions for the existence of solutions of a class of $n$ th-order linear boundary value problems, in the form of integral inequalities. Such a modification simplifies the assessment of the conditions on restricting the inequality to be verified to a single point instead of the full interval where the boundary value problem is defined. The paper also provides an error bound that needs to be considered in the integral inequalities of the previous paper when they are calculated numerically.

\section{Introduction}

Let $I$ be a compact interval in $\mathbb{R}$, let $k, n \in \mathbb{N}$ be such that $1 \leq k<n$, and let us consider the $n$ th-order boundary value problem:

$$
\begin{aligned}
& L y\left.=\sum_{i=0}^{\mu} c_{i}(x) y^{(i)}(x), \quad x \in\right] a^{\prime}, b^{\prime}[, \\
& y^{(i)}\left(a^{\prime}\right)=0, \quad i=0,1,2, \ldots, k-1, \\
& y^{\left(\beta_{i}\right)}\left(b^{\prime}\right)=0, \\
& i=1,2, \ldots, n-k, 0 \leq \beta_{1}<\beta_{2}<\cdots<\beta_{n-k}<n-1,
\end{aligned}
$$

where $\left[a^{\prime}, b^{\prime}\right] \quad \subset \quad I$ and $c_{j}(x)$ are functions piecewise continuous on $I$ such that

$$
\begin{aligned}
& (-1)^{n-k} c_{j}(x) \geq 0, \quad x \in I, 0 \leq j \leq \mu, \\
& (-1)^{n-k} c_{l}(x)>0 \quad \text { a.e. on } I
\end{aligned}
$$

for at least one $l$,

$$
0 \leq \mu \leq \beta_{1} \text {, }
$$

and $L: C^{n}(I) \rightarrow C(I)$ is the right-disfocal differential operator defined by

$$
\begin{array}{r}
L y=y^{(n)}(x)+a_{n-1}(x) y^{(n-1)}(x)+\cdots+a_{0}(x) y(x), \\
x \in I,
\end{array}
$$

with $a_{j}(x) \in C(I), 0 \leq j \leq n-1$. Examples of these types of problems appear in the study of the deflections of beams, both straight ones with nonhomogeneous cross sections in free vibration, which are subject to the fourth-order linear EulerBernoulli equation, and curved ones with different shapes. An account of these and other applications can be found in [1, Chapter IV].

In a recent paper of the authors (see [2, Theorems 8,9 and 10]) it was shown that the recursive application of the operator $M: C^{\mu}[a, b] \rightarrow C^{n}[a, b]$ is defined by

$$
M f=\int_{a}^{b} G(x, t) \sum_{i=0}^{\mu} c_{i}(t) f^{(i)}(t) d t
$$


where $[a, b] \subset I$ and $G(x, t)$ is the Green function of the problem:

$$
\begin{aligned}
& L y=0, \\
& y^{(i)}(a)=0, \quad i=0,1,2, \ldots, k-1, \\
& y^{\left(\beta_{i}\right)}(b)=0, \\
& i=1,2, \ldots, n-k, 0 \leq \beta_{1}<\beta_{2}<\cdots<\beta_{n-k}<n-1,
\end{aligned}
$$

to functions $y$ belonging to a cone $P$ with some properties, providing a procedure to determine the existence (or not) of solutions of the problem (1)-(6) for $a^{\prime}$ and $b^{\prime}$ interior to $a, b$ (i.e., for $a^{\prime}, b^{\prime}$ satisfying $a<a^{\prime}<b^{\prime} \leq b$ or $a \leq a^{\prime}<b^{\prime}<b$ ). The procedure was based on the evaluation of whether either $M^{j} y-M^{i} y$ or $M^{i} y-M^{j} y$ belonged to the cone $P$ for different values of $j>i \geq 0$. Such an evaluation implied in practice the comparison of the functions $\partial^{\beta_{1}} M^{j} y / \partial x^{\beta_{1}}$ and $\partial^{\beta_{1}} M^{i} y / \partial x^{\beta_{1}}$ over the full interval $[a, b]$, so that

(i) if $(-1)^{n-k}\left(\partial^{\beta_{1}} M^{j} y / \partial x^{\beta_{1}}\right) \leq(-1)^{n-k}\left(\partial^{\beta_{1}} M^{i} y / \partial x^{\beta_{1}}\right)$ for all $x \in[a, b]$, then there was no solution of (1) satisfying (2)-(3) at extremes interior to $a, b$;

(ii) if $(-1)^{n-k}\left(\partial^{\beta_{1}} M^{j} y / \partial x^{\beta_{1}}\right) \geq(-1)^{n-k}\left(\partial^{\beta_{1}} M^{i} y / \partial x^{\beta_{1}}\right)$ for all $x \in[a, b]$, then there was a solution of (1) satisfying (2)-(3) either at $a, b$ or at extremes interior to $a, b$.

The iterative comparison yielded lower and upper bounds for the extremes $a^{\prime}$ and $b^{\prime}$ for which (1)-(3) has a nontrivial solution, bounds which converge to the values of these extremes $a^{\prime}$ and $b^{\prime}$ as the recursivity index grows.

One of the few drawbacks of the method of [2] is that when the calculation of $\partial^{\beta_{1}} M^{j} y / \partial x^{\beta_{1}}$ is done numerically using a partition $\left\{x_{l}\right\}$ of $[a, b]$, very often such a calculation only yields values at the points $\left\{x_{l}\right\}$ and not at the interior points of each subinterval $\left[x_{l}, x_{l+1}\right]$. Since the aforementioned inequalities have to be satisfied for all $x \in[a, b]$, in these cases we need to introduce a security margin $\delta>0$ in the comparison of the discrete values $\partial^{\beta_{1}} M^{j} y\left(x_{l}\right) / \partial x^{\beta_{1}}$ and $\partial^{\beta_{1}} M^{i} y\left(x_{l}\right) / \partial x^{\beta_{1}}$ for all $l$ (i.e., to enforce that either $(-1)^{n-k}\left(\partial^{\beta_{1}} M^{j} y\left(x_{l}\right) / \partial x^{\beta_{1}}\right)+\delta \leq(-1)^{n-k}\left(\partial^{\beta_{1}} M^{i} y\left(x_{l}\right) / \partial x^{\beta_{1}}\right)$ or $\left.(-1)^{n-k}\left(\partial^{\beta_{1}} M^{j} y / \partial x^{\beta_{1}}\right) \geq(-1)^{n-k}\left(\partial^{\beta_{1}} M^{i} y / \partial x^{\beta_{1}}\right)+\delta\right)$ in a manner that guarantees that the inequalities hold for all $x \in[a, b]$.

With this constraint in mind, the purpose of this paper is twofold:

(i) To calculate such a security margin $\delta$ that, once taken into account when comparing the mentioned functions over the set $\left\{x_{l}\right\}$, ensures that the same results are obtained on the full interval $[a, b]$.

(ii) To show that under certain conditions (namely, $\mu<$ $\beta_{1}$; compare with the original $\mu \leq \beta_{1}$ of [2]) and upon selection of the proper functions $y$, it is possible to restrict the comparison of derivatives of $M^{j} y$ and $M^{i} y$ to a specific point in $[a, b]$ instead of the full interval. The argument inspires on an idea from Keener for the focal problem (see [3]).
In terms of nomenclature, we will use the notation $M$ to name the operator defined in (7), $M f$ or $M\{f\}$ to name the function with domain $[a, b]$ resulting from the application of $M$ to $f(x) \in C^{\mu}[a, b], M^{i} f$ or $M^{i}\{f\}$ to name the function with domain $[a, b]$ resulting from the application of $M$ to $f(x) \in C^{\mu}[a, b]$ recursively $i$ times, and $M f(x)$ to name the value of the function $M f$ at the point $x$. We will use $M_{a b}$ when we want to stress the dependence of $M$ with the extremes where it is defined. And we will denote by $P C[a, b]$ the set of piecewise continuous functions on $[a, b]$.

In order to make this paper self-contained, let us recall that, given a Banach space $B$, a cone $P \subset B$ is a nonempty closed set defined by the following conditions:

(1) If $u, v \in P$, then $c u+d v \in P$ for any real numbers $c, d \geq 0$.

(2) If $u \in P$ and $-u \in P$, then $u=0$.

We will denote the interior of the cone $P$ by $P^{0}$, and we will say that the cone $P$ is reproducing if any $y \in B$ can be expressed as $y=u-v$ with $u, v \in P$. The existence of a cone in a Banach space $B$ allows defining a partial ordering relationship in that Banach space by setting $u \leq v$ if and only if $v-u \in P$. Accordingly, we will say that the operator $M$ is $u_{0}$-positive if there exists a $u_{0} \in P$ such that for any $v \in P \backslash\{0\}$ one can find positive constants $\delta_{1}$ and $\delta_{2}$ such that $\delta_{1} u_{0} \leq M v \leq \delta_{2} u_{0}$ (note that the constants $\delta_{1}$ and $\delta_{2}$ do not need to be the same for all $v$ ). We will denote by $r(M)$ the spectral radius of $M$ (in other words, $r(M)$ is the supremum of the spectrum of $M$ ).

The main result of [2] is Theorem 2, which we will state also here for completeness.

Theorem 1 (see [2, Theorem 2]). Let us suppose that there is a Banach space $B$ and a reproducing cone $P$ therein for which $M(P) \subset P$ and $M$ is $u_{0}$-positive. Then the eigenvalue problem $M u=\lambda u$ has a solution $u \in P$ and its associated eigenvalue $\lambda$ is positive, simple, and bigger in absolute value than any other eigenvalue of such a problem.

In addition, if $r(M)$ is strictly increasing with the length of the interval $[a, b]$ (i.e., if $a$ is fixed, $r\left(M_{a b}\right)$ is increasing with $b$ and if $b$ is fixed, $r\left(M_{a b}\right)$ is decreasing with $\left.a\right)$ and $\lim _{b \rightarrow a^{+}} r\left(M_{a b}\right)=\lim _{a \rightarrow b^{-}} r\left(M_{a b}\right)=0$, one has the following:

(i) If there is no nontrivial solution of (1)-(6) at extremes $a^{\prime}, b^{\prime}$ equal or interior to $a, b$, then

$$
\lim _{k \rightarrow \infty} M^{k} v=0
$$

for any $v \in P$ and for any $v, w \in P \backslash\{0\}$ there exists $k_{0} \geq 1$ such that

$$
M^{k} v \leq M w, \quad k \geq k_{0}
$$

and there cannot be any $v \in P \backslash\{0\}$ and any $k_{1} \geq 1$ such that

$$
M^{k_{1}} v \geq v
$$


(ii) If there is a nontrivial solution of (1)-(6) at extremes $a^{\prime}, b^{\prime}$ interior to $a, b$, then for any $v, w \in P \backslash\{0\}$ there exists a $k_{2} \geq 1$ such that

$$
M^{k} v \geq M w, \quad k \geq k_{2},
$$

and there cannot be any $v \in P \backslash\{0\}$ and any $k_{3} \geq 1$ such that

$$
M^{k_{3}} v \leq v
$$

From the historical point of view, let us remark that the use of the theory of cones in boundary value problems dates from the works of Krein and Rutman [4] and Krasnosel'skii [5], which were continued by multiple authors. References [3, 6-20] are a good account of this.

The organization of the paper is as follows. Section 2 will describe a new method to obtain necessary and sufficient conditions for the problem (1)-(6) to have a nontrivial solution, which restricts the comparison of functions to one point. In Section 3 the security margin that needs to be considered in the application of [2, Theorems 8-10] will be calculated using linear splines theory. Section 4 will apply the previous results to several examples. Finally Section 5 will provide some conclusions.

\section{A Cone and a Procedure to Reduce the Comparison of Functions to One Point}

In this section we will first define a new cone different from that used in [2, Theorem 8] and will prove that it satisfies the properties required by Theorem 1 . Then we will show that, for some specific functions $y$ belonging to that cone, the application of Theorem 1 implies a comparison of the values of a certain derivative of $M^{j} y$ and $y$ at a single point in $[a, b]$.

Thus, let us consider the eigenvalue problem

$$
M u(x)=\lambda u(x), \quad x \in[a, b],
$$

with $M$ defined as in (7). Let us define the Banach space $B$ as

$$
B=\{y \in P C[a, b]\},
$$

if $\mu=0$, and as

$$
\begin{aligned}
B & =\left\{y \in C^{\mu-1}[a, b]: y^{(\mu)}(x) \in P C[a, b], y^{(i)}(a)\right. \\
& =0,0 \leq i \leq \mu-1\},
\end{aligned}
$$

if $\mu>0$; in both cases the associated norm is

$$
\begin{aligned}
& \|y\| \\
& \quad=\max \left\{\sup \left\{\left|y^{(i)}(x)\right|, x \in[a, b]\right\}, i=0, \ldots, \mu\right\} .
\end{aligned}
$$

Let us also define the cone $P$ by

$$
P=\left\{y \in B:(-1)^{n-k} y^{(\mu)}(x) \geq 0 ; x \in[a, b]\right\} .
$$

From the definition of $P$ it is clear that $(-1)^{n-k} y^{(i)}(x) \geq 0, x \in$ $[a, b], 0 \leq i \leq \mu$. With the help of the cone $P$ it is possible to prove the following theorem.
Theorem 2. The conclusions of Theorem 1 are applicable to the problem (1)-(6) and the cone P defined in (18).

Proof. We only need to prove that $P$ is a reproducing cone, that $M(P) \subset P$, and that $M$ is $u_{0}$-positive in $P$, as this guarantees the existence of an eigenfunction $u \in P$ with a positive maximal eigenvalue $\lambda$ (e.g., see [3, Theorem 2.1]) and both the monotonicity of this eigenvalue with the extremes $a$ and $b$ and the compacticity of $M$ were already proven in [2, Theorem 8].

Thus, using the notation

$$
\begin{aligned}
& \{u(x)\}^{+}=(-1)^{n-k} \max \left\{(-1)^{n-k} u(x), 0\right\}, \\
& \{u(x)\}^{-}=(-1)^{n-k} \max \left\{-(-1)^{n-k} u(x), 0\right\},
\end{aligned}
$$

for $x \in[a, b]$, it is clear that

$$
y^{(\mu)}(x)=\left\{y^{(\mu)}(x)\right\}^{+}-\left\{y^{(\mu)}(x)\right\}^{-}, \quad x \in[a, b] .
$$

If $\mu=0$ the reproducing character of $P$ is immediate. Otherwise, we can get to the same conclusion by noting that

$$
\begin{aligned}
y(x)= & \int_{a}^{x} \frac{(x-t)^{\mu-1}}{(\mu-1) !}\left\{y^{(\mu)}(t)\right\}^{+} d t \\
& -\int_{a}^{x} \frac{(x-t)^{\mu-1}}{(\mu-1) !}\left\{y^{(\mu)}(t)\right\}^{-} d t, \quad x \in[a, b] ;
\end{aligned}
$$

for any $y \in B$, the two terms of the right-hand side of (21) being functions which belong to $P$.

To prove the $u_{0}$-positivity of $M$, let us consider the same auxiliar Banach space $\mathscr{B}$ defined in [2, Theorem 8]; namely,

$$
\begin{aligned}
\mathscr{B} & =\left\{y \in C^{n-1}[a, b]: y^{(i)}(a)=0,0 \leq i \leq k\right. \\
& \left.-1 ; y^{\left(\beta_{i}\right)}(b)=0, \quad 1 \leq i \leq n-k\right\},
\end{aligned}
$$

and the auxiliar cone $\mathscr{P}$ defined by

$$
\mathscr{P}=\left\{y \in \mathscr{B}:(-1)^{n-k} y^{\left(\beta_{1}\right)}(x) \geq 0, x \in[a, b]\right\}
$$

whose interior, if we denote by $m$ the lowest integer that satisfies $m>\beta_{1}$ and $m \neq \beta_{2}, \ldots, \beta_{n-k}$, is given by

$$
\begin{aligned}
\mathscr{P}^{0} & =\left\{y \in \mathscr{B}:(-1)^{n-k} y^{\left(\beta_{1}\right)}(x)>0, x\right. \\
& \in] a, b\left[;(-1)^{n-k} y^{(k)}(a)\right. \\
& \left.>0 ;(-1)^{n-k-m+\beta_{1}} y^{(m)}(b)>0\right\} .
\end{aligned}
$$

Following exactly the same reasoning used in [2, Theorem 8] and using hypotheses (4) and (15)-(18) it is straightforward to prove that $M$ maps $P \backslash\{0\}$ into $\mathscr{P}^{0}$, that is, $M v \in \mathscr{P}^{0}$ for any $v \in P \backslash\{0\}$. Since $\mathscr{P} \subset P$ (this follows from the facts that $\mathscr{B} \subset$ $B$ and $\left.\mu \leq \beta_{1}\right)$, one has that $M(P) \subset P$, and also that for any $u_{0} \in \mathscr{P}^{0}$ there must be an $\epsilon_{1}>0$ such that

$$
M v-\epsilon_{1} u_{0} \in \mathscr{P}
$$


which implies

$$
\left.M v \geq \epsilon_{1} u_{0} \quad \text { (w.r.t. } \mathscr{P}\right) .
$$

Likewise, there must be an $\epsilon_{2}>0$ such that

$$
u_{0}-\epsilon_{2} M v \in \mathscr{P}
$$

which implies

$$
\left.u_{0} \geq \epsilon_{2} M v \quad \text { (w.r.t. } \mathscr{P}\right) .
$$

Combining (26) and (28) one gets

$$
\left.\epsilon_{1} u_{0} \leq M v \leq \frac{u_{0}}{\epsilon_{2}} \quad \text { (w.r.t. } \mathscr{P}\right) .
$$

As $u_{0} \in \mathscr{P}^{0} \subset \mathscr{P} \subset P$, (29) proves that $M$ is $u_{0}$-positive in $P$. This completes the proof.

The next theorem will allow us to exploit Theorem 2 as explained in Section 1.

Theorem 3. Let us suppose that $\mu<\beta_{1}$ and that $w \in P \backslash\{0\}$ is such that $w^{(\mu+1)}(x)$ exists and satisfies $(-1)^{n-k} w^{(\mu+1)}(x) \leq 0$ on those points of $[a, b]$ where $w^{(\mu)}(x)$ is continuous. One has the following:

(1) If $w(x) \in C^{\mu}[a, b]$ and there exists an integer $j>0$ such that

$$
(-1)^{n-k} w^{(\mu)}(b) \geq(-1)^{n-k} \frac{\partial^{\mu} M^{j} w(b)}{\partial x^{\mu}},
$$

then the problem (1)-(6) cannot have a nontrivial solution at extremes $a^{\prime}, b^{\prime}$ interior to $a, b$.

(2) If $(-1)^{n-k} w^{(\mu)}(a) \leq 0$, there exists a single discontinuity point $z \in[a, b]$ of $w^{(\mu)}(x)$ such that

$$
\begin{aligned}
\lim _{x \rightarrow z^{-}}(-1)^{n-k} w^{(\mu)}(x) & <(-1)^{n-k} w^{(\mu)}(z) \\
& =\lim _{x \rightarrow z^{+}}(-1)^{n-k} w^{(\mu)}(x),
\end{aligned}
$$

and there exists an integer $j>0$ such that

$$
(-1)^{n-k} w^{(\mu)}(z) \leq(-1)^{n-k} \frac{\partial^{\mu} M^{j} w(z)}{\partial x^{\mu}},
$$

then the problem (1)-(6) does have a nontrivial solution either at $a, b$ or at extremes $a^{\prime}, b^{\prime}$ interior to $a, b$.

Proof. If $(-1)^{n-k} w^{(\mu+1)}(x) \leq 0$ at those points of $[a, b]$ where $w^{(\mu)}(x)$ is continuous, then from [21, Theorem 2.1], the function defined by

$$
v(x)=w(x)-M^{j} w(x), \quad x \in[a, b]
$$

satisfies

$$
(-1)^{n-k} v^{(\mu+1)}(x)<0,
$$

at those points of $] a, b\left[\right.$ where $w^{(\mu)}(x)$ is continuous.

Thus, from the hypotheses of statement (1), one has that $v(x) \in C^{\mu}[a, b]$ and $(-1)^{n-k} v^{(\mu)}(b) \geq 0$, which together with (34) yield

$$
(-1)^{n-k} v^{(\mu)}(x) \geq 0, \quad x \in[a, b]
$$

that is, $w \geq M^{j} w$ with regard to the cone $P$. From Theorems 1 and 2 one gets the first conclusion.

On the other hand, from the hypotheses of statements (2), (2), (7), and (34), one has that $(-1)^{n-k} v^{(\mu)}(a) \leq 0$, that $(-1)^{n-k} v^{(\mu)}(x)$ is decreasing on $[a, b]$ except at the point $x=z$, where it has a discontinuity jump, and that $(-1)^{n-k} v^{(\mu)}(z) \leq 0$. This implies that

$$
(-1)^{n-k} v^{(\mu)}(x) \leq 0, \quad x \in[a, b],
$$

which in turn implies that $w \leq M^{j} w$ with regard to the cone $P$. Again, from Theorems 1 and 2, one gets the second conclusion.

Remark 4. It is possible to extend the results of Theorems 2 and 3 to cones similar to $P$ where the condition $(-1)^{n-k} v^{(\mu)}(x) \geq 0$ is replaced by $(-1)^{n-k} v^{(l)}(x) \geq 0$, as long as $\mu \leq l<\beta_{1}$, since in that case $(-1)^{n-k}\left(\partial^{l+1} G(x, t) / \partial x^{l+1}\right) \geq$ 0 for $\mu \leq l<\beta_{1}$ (this follows from [21, Theorem 2.1], as mentioned in the proof), which implies $(-1)^{n-k}\left(\partial^{l+1} M^{j} v(x) / \partial x^{l+1}\right) \geq 0$ for those $v \in P$. This latter fact is indeed the key that allows restricting the comparison of functions to one point in Theorem 3.

To apply statement (1) of Theorem 3 we can consider, for instance, the function

$$
w(x)=(-1)^{n-k} \frac{(x-a)^{\mu}}{\mu !}, \quad x \in[a, b],
$$

which satisfies

$$
\begin{aligned}
w^{(\mu)}(x) & =(-1)^{n-k}, \\
w^{(\mu+1)}(x) & =0, \\
x \in[a, b] ; & \\
w(a) & =w^{\prime}(a)=\cdots=w^{(\mu-1)}(a)=0 .
\end{aligned}
$$

And in order to apply statement (2) of Theorem 3 we can pick any $z \in] a, b[$ and define, for example, the function

$$
w(x)= \begin{cases}0, & a \leq x<z, \\ (-1)^{n-k} \frac{(x-z)^{\mu}}{\mu !}, & z \leq x \leq b,\end{cases}
$$


which satisfies $w^{(i)}(a)=0$ for $0 \leq i \leq \mu-1$,

$$
w^{(\mu)}(x)= \begin{cases}0, & a \leq x<z, \\ (-1)^{n-k}, & z \leq x \leq b,\end{cases}
$$

and $w^{(\mu+1)}(x)=0$ on $[a, z[\cup] z, b]$.

Remark 5. As pointed out in [2], (1)-(6) is just a way of representing a set of problems of the type

$$
\begin{array}{r}
y^{(n)}(x)+p_{n-1}(x) y^{(n-1)}(x)+\cdots+p_{0}(x) y(x)=0, \\
x \in[a, b] ; \\
y^{(i)}(a)=0, \\
i=0,1,2, \ldots, k-1, \\
y^{\left(\beta_{i}\right)}(b)=0, \\
i=1,2, \ldots, n-k, 0 \leq \beta_{1}<\beta_{2}<\cdots<\beta_{n-k}<n-1,
\end{array}
$$

in a way that allows the existence and calculation of the Green function of the problem $L y=0$ with boundary conditions (42)-(43), while guaranteeing the right disfocality of $L y=0$ on $[a, b]$ and at the same time yielding functions $c_{i}(x), 0 \leq$ $i \leq \mu$, which satisfy the conditions for the application of the method. But there still exists some freedom in the choice of $a_{i}(x)$ and $c_{i}(x)$ as long as $p_{i}(x)=a_{i}(x)-c_{i}(x), 0 \leq i \leq \mu$, so that it is normal to wonder what choices of such functions give better results than others. [2, Theorem 12] and [2, Remark 13] answered that question for the problem (1)-(6) and a cone different from (18) by proving the faster convergence of the method when the functions $c_{i}(x), 0 \leq i \leq \mu$ were closer to zero. It is easy to prove that such a behaviour is also applicable to the cone $P$ defined in (18) taking into account the fact that $M$ maps $P$ into the cone $\mathscr{P}$ of $(23)$.

As was done in [2, Theorem 11 and Remark 15], it is possible to establish theorems similar to Theorems 2 and 3 for the problem symmetric to (1)-(6) and defined by

$$
\begin{aligned}
L y & =\sum_{i=0}^{\mu} c_{i}(x) y^{(i)}(x), \quad x \in\left[a^{\prime}, b^{\prime}\right], \\
y^{\left(\alpha_{i}\right)}\left(a^{\prime}\right) & =0, \\
i & =1,2, \ldots, k, 0 \leq \alpha_{1}<\alpha_{2}<\cdots<\alpha_{k}<n-1, \\
y^{(i)}\left(b^{\prime}\right) & =0, \quad i=0,1,2, \ldots, n-k-1,
\end{aligned}
$$

with $\left[a^{\prime}, b^{\prime}\right] \subset I, L$ defined as in (6) and left disfocal on $I$,

$$
\begin{gathered}
0 \leq \mu<\alpha_{1}, \\
(-1)^{n-k-i} c_{i}(x) \geq 0, \quad x \in I, \quad 0 \leq i \leq \mu, \\
(-1)^{n-k-j} c_{j}(x)>0 \quad \text { a.e. on } I
\end{gathered}
$$

for at least one $j$ such that $0 \leq j \leq \mu$. To that end one needs to consider the interval $[a, b] \subset I$ and use the Banach spaces

$$
B=\{y \in P C[a, b]\},
$$

if $\mu=0$, and

$$
\begin{aligned}
B & =\left\{y \in C^{\mu-1}[a, b]: y^{(\mu)}(x) \in P C[a, b] ; y^{(i)}(b)\right. \\
& =0,0 \leq i \leq \mu-1\},
\end{aligned}
$$

if $\mu>0$, associated with the norm

$$
\begin{aligned}
& \|y\| \\
& \quad=\max \left\{\sup \left\{\left|y^{(i)}(x)\right|, x \in[a, b]\right\}, i=0, \ldots, \mu\right\},
\end{aligned}
$$

as well as the cone

$$
P=\left\{y \in B:(-1)^{n-k-\mu} y^{(\mu)}(x) \geq 0 ; x \in[a, b]\right\} .
$$

Since the reasoning to prove them is the same as that used in Theorems 2 and 3, we will state the equivalent theorems without proof.

Theorem 6. The conclusions of Theorem 1 are applicable to the problem (44)-(45) and the cone $P$ defined in (49).

Theorem 7. Let us suppose that $w \in P \backslash\{0\}$ such that $w^{(\mu+1)}(x)$ exists and satisfies $(-1)^{n-k-\mu} w^{(\mu+1)}(x) \geq 0$ on those points of $[a, b]$ where $w^{(\mu)}(x)$ is continuous. One has the following:

(1) If $w(x) \in C^{\mu}[a, b]$ and there exists an integer $j>0$ such that

$$
(-1)^{n-k-\mu} w^{(\mu)}(a) \geq(-1)^{n-k-\mu} \frac{\partial^{\mu} M^{j} w(a)}{\partial x^{\mu}},
$$

then the problem (44)-(45) cannot have a nontrivial solution at extremes $a^{\prime}, b^{\prime}$ interior to $a, b$.

(2) If $(-1)^{n-k-\mu} w^{(\mu)}(b) \leq 0$, there exists a single discontinuity point $z \in[a, b]$ of $w^{(\mu)}(x)$ such that

$$
\begin{gathered}
\lim _{x \rightarrow z^{-}}(-1)^{n-k-\mu} w^{(\mu)}(x)=(-1)^{n-k-\mu} w^{(\mu)}(z) \\
>\lim _{x \rightarrow z^{+}}(-1)^{n-k-\mu} w^{(\mu)}(x),
\end{gathered}
$$

and there exists an integer $j>0$ such that

$$
(-1)^{n-k-\mu} w^{(\mu)}(z) \leq(-1)^{n-k-\mu} \frac{\partial^{\mu} M^{j} w(z)}{\partial x^{\mu}},
$$

then the problem (44)-(45) does have a nontrivial solution either at $a, b$ or at extremes $a^{\prime}, b^{\prime}$ interior to $a, b$

A function which satisfies the conditions of the statement (1) of Theorem 7 is, for instance,

$$
w(x)=(-1)^{n-k} \frac{(b-x)^{\mu}}{\mu !}, \quad x \in[a, b],
$$


which verifies

$$
\begin{aligned}
w^{(\mu)}(x)=(-1)^{n-k-\mu}, & \\
w^{(\mu+1)}(x)=0, & x \in[a, b] ; \\
w(b)=w^{\prime}(b)=\cdots=w^{(\mu-1)}(b)=0 . &
\end{aligned}
$$

And a candidate function which fulfils the conditions of the statement (2) of Theorem 7 is

$$
w(x)= \begin{cases}(-1)^{n-k} \frac{(z-x)^{\mu}}{\mu !}, & a \leq x \leq z, \\ 0, & z<x \leq b,\end{cases}
$$

for any $z \in] a, b\left[\right.$, which satisfies $w^{(i)}(b)=0$ for $0 \leq i \leq \mu-1$,

$$
w^{(\mu)}(x)= \begin{cases}(-1)^{n-k-\mu}, & a \leq x \leq z, \\ 0, & z<x \leq b,\end{cases}
$$

and $w^{(\mu+1)}(x)=0$ on $[a, z[\cup] z, b]$.

\section{Calculation of the Security Margin for the General Case of [2, Theorem 8]}

As mentioned in Section 1, the application of [2, Theorems 8$10]$ to the problem (1)-(6) requires the determination of the sign of the function $\partial^{\beta_{1}} M^{j} y(x) / \partial x^{\beta_{1}}-\partial^{\beta_{1}} M^{i} y(x) / \partial x^{\beta_{1}}$ for $j>i \geq 0$ and $y(x)$ belonging to the cone $P$ of $[2$, equation (55)], so that

(i) if the sign of such a function over $[a, b]$ is the opposite to that of $(-1)^{n-k}$, then there is no solution of (1) satisfying (2)-(3) at extremes interior to $a, b$;

(ii) if the sign of such a function over $[a, b]$ is the same as that of $(-1)^{n-k}$, then there is a solution of (1) satisfying (2)-(3) either at $a, b$ or at extremes interior to $a, b$.

With this in mind, this section aims at answering the question of what security margin to consider in the evaluation of $\partial^{\beta_{1}} M^{j} y(x) / \partial x^{\beta_{1}}-\partial^{\beta_{1}} M^{i} y(x) / \partial x^{\beta_{1}}$ at a partition $\left\{x_{l}\right\}$ of $[a, b]$ for $j>i \geq 0$, in order to guarantee that the sign of that function holds for all $x \in[a, b]$. It is, therefore, a problem of assessing the value of $\partial^{\beta_{1}} M^{j} y(x) / \partial x^{\beta_{1}}-\partial^{\beta_{1}} M^{i} y(x) / \partial x^{\beta_{1}}$ in each subinterval $\left[x_{l}, x_{l+1}\right]$ based on the knowledge that we have of $\partial^{\beta_{1}} M^{j} y\left(x_{l}\right) / \partial x^{\beta_{1}}-\partial^{\beta_{1}} M^{i} y\left(x_{l}\right) / \partial x^{\beta_{1}}$ for all $l$, a problem of interpolation.

To this end, given the partition $\left\{x_{l}\right\}$ of $[a, b]$ and a function $f(x) \in C[a, b]$, let us recall the concept of a linear spline (see [22, Sections 6.1 and 6.2]), defined as a function $s(x) \in C[a, b]$ which is a linear polynomial in each of the subintervals $\left[x_{l}, x_{l+1}\right]$ and takes the value $f\left(x_{l}\right)$ at each of the points $\left\{x_{l}\right\}$. Numerically that can be expressed as

$$
\begin{aligned}
s(x)=f\left(x_{l+1}\right) \frac{x-x_{l}}{x_{l+1}-x_{l}}+f\left(x_{l}\right) \frac{x_{l+1}-x}{x_{l+1}-x_{l}}, & \\
x & \in\left[x_{l}, x_{l+1}\right] .
\end{aligned}
$$

Applying (57) to $\partial^{\beta_{1}} M^{j} y(x) / \partial x^{\beta_{1}}-\partial^{\beta_{1}} M^{i} y(x) / \partial x^{\beta_{1}}$ one gets the spline

$$
\begin{array}{r}
s(x)=\left\{\frac{\partial^{\beta_{1}} M^{j} y\left(x_{l+1}\right)}{\partial x^{\beta_{1}}}-\frac{\partial^{\beta_{1}} M^{i} y\left(x_{l+1}\right)}{\partial x^{\beta_{1}}}\right\} \frac{x-x_{l}}{x_{l+1}-x_{l}} \\
+\left\{\frac{\partial^{\beta_{1}} M^{j} y\left(x_{l}\right)}{\partial x^{\beta_{1}}}-\frac{\partial^{\beta_{1}} M^{i} y\left(x_{l}\right)}{\partial x^{\beta_{1}}}\right\} \frac{x_{l+1}-x}{x_{l+1}-x_{l}}, \\
x \in\left[x_{l}, x_{l+1}\right] .
\end{array}
$$

The spline $s(x)$ of (58) interpolates the function $\partial^{\beta_{1}} M^{j} y(x) /$ $\partial x^{\beta_{1}}-\partial^{\beta_{1}} M^{i} y(x) / \partial x^{\beta_{1}}$ with an interpolation error $e(x)$; that is,

$$
\frac{\partial^{\beta_{1}} M^{j} y(x)}{\partial x^{\beta_{1}}}-\frac{\partial^{\beta_{1}} M^{i} y(x)}{\partial x^{\beta_{1}}}=s(x)+e(x),
$$

$$
x \in[a, b] .
$$

If $\partial^{\beta_{1}} M^{j} y(x) / \partial x^{\beta_{1}}-\partial^{\beta_{1}} M^{i} y(x) / \partial x^{\beta_{1}} \in C^{2}[a, b]$, from [22, Section 6.5.1], one gets the fact that this interpolation error can be calculated in each subinterval $\left[x_{l}, x_{l+1}\right]$ as

$$
\begin{aligned}
e(x) & =\frac{\left(x-x_{l}\right)\left(x_{l+1}-x\right)}{2}\left\{\frac{\partial^{\beta_{1}+2} M^{j} y(\xi)}{\partial x^{\beta_{1}+2}}\right. \\
& \left.-\frac{\partial^{\beta_{1}+2} M^{i} y(\xi)}{\partial x^{\beta_{1}+2}}\right\},
\end{aligned}
$$

where $\xi$ is a value in $\left[x_{l}, x_{l+1}\right]$ which depends on $x$. Maximizing the right-hand side of (60) one yields

$$
\begin{aligned}
& |e(x)| \leq \frac{\left(x_{l+1}-x_{l}\right)^{2}}{8} \\
& \quad \max \left\{\left|\frac{\partial^{\beta_{1}+2} M^{j} y(\xi)}{\partial x^{\beta_{1}+2}}-\frac{\partial^{\beta_{1}+2} M^{i} y(\xi)}{\partial x^{\beta_{1}+2}}\right|, \xi\right. \\
& \left.\quad \in\left[x_{l}, x_{l+1}\right]\right\} .
\end{aligned}
$$

From (59) and (61) one gets the following theorem, which gives the searched security margin.

Theorem 8. If $\partial^{\beta_{1}} M^{j} y\left(x_{l}\right) / \partial x^{\beta_{1}}-\partial^{\beta_{1}} M^{i} y\left(x_{l}\right) / \partial x^{\beta_{1}}$ and $\partial^{\beta_{1}} M^{j} y\left(x_{l+1}\right) / \partial x^{\beta_{1}}-\partial^{\beta_{1}} M^{i} y\left(x_{l+1}\right) / \partial x^{\beta_{1}}$ have the same sign for some $j>i \geq 0$ and

$$
\begin{aligned}
& \min \left\{\left|\frac{\partial^{\beta_{1}} M^{j} y\left(x_{l}\right)}{\partial x^{\beta_{1}}}-\frac{\partial^{\beta_{1}} M^{i} y\left(x_{l}\right)}{\partial x^{\beta_{1}}}\right|,\right. \\
& \left.\left|\frac{\partial^{\beta_{1}} M^{j} y\left(x_{l+1}\right)}{\partial x^{\beta_{1}}}-\frac{\partial^{\beta_{1}} M^{i} y\left(x_{l+1}\right)}{\partial x^{\beta_{1}}}\right|\right\} \geq \frac{\left(x_{l+1}-x_{l}\right)^{2}}{8} \\
& \cdot \max \left\{\left|\frac{\partial^{\beta_{1}+2} M^{j} y(\xi)}{\partial x^{\beta_{1}+2}}-\frac{\partial^{\beta_{1}+2} M^{i} y(\xi)}{\partial x^{\beta_{1}+2}}\right|, \xi\right. \\
& \left.\in\left[x_{l}, x_{l+1}\right]\right\},
\end{aligned}
$$


then the sign of $(-1)^{n-k}\left(\partial^{\beta_{1}} M^{j} y(x) / \partial x^{\beta_{1}}-\partial^{\beta_{1}} M^{i} y(x) / \partial x^{\beta_{1}}\right)$ is the same as the sign of $(-1)^{n-k}\left(\partial^{\beta_{1}} M^{j} y\left(x_{l}\right) / \partial x^{\beta_{1}}-\partial^{\beta_{1}} M^{\mathrm{i}} y\left(x_{l}\right)\right.$ / $\left.\partial x^{\beta_{1}}\right)$ for all $x \in\left[x_{l}, x_{l+1}\right]$.

In order to apply Theorem 8 we need to calculate a bound for $\partial^{\beta_{1}+2} M^{j} y(\xi) / \partial x^{\beta_{1}+2}-\partial^{\beta_{1}+2} M^{i} y(\xi) / \partial x^{\beta_{1}+2}$ on $\left[x_{l}, x_{l+1}\right]$. If $\beta_{1} \leq n-3$, from (7), we know that such a function can be obtained as

$$
\begin{aligned}
& \frac{\partial^{\beta_{1}+2} M^{j} y(\xi)}{\partial x^{\beta_{1}+2}}-\frac{\partial^{\beta_{1}+2} M^{i} y(\xi)}{\partial x^{\beta_{1}+2}} \\
& =\int_{a}^{b} \frac{\partial^{\beta_{1}+2} G(\xi, t)}{\partial x^{\beta_{1}+2}} \sum_{m=0}^{\mu} c_{m}(t) \\
& \cdot\left(\frac{\partial^{m} M^{j-1} y(t)}{\partial x^{m}}-\frac{\partial^{m} M^{i-1} y(t)}{\partial x^{m}}\right) d t .
\end{aligned}
$$

Otherwise, such a calculation can be done by taking into account, from (6), (7), and (8), that

$$
\begin{aligned}
L M^{i} y(x)= & \frac{\partial^{n} M^{i} y(x)}{\partial x^{n}}+a_{n-1}(x) \frac{\partial^{n-1} M^{i} y(x)}{\partial x^{n-1}}+\cdots \\
& +a_{0}(x) M^{i} y(x) \\
= & \sum_{m=0}^{\mu} c_{m}(x) \frac{\partial^{m} M^{i-1} y(x)}{\partial x^{m}} .
\end{aligned}
$$

However, if we are calculating $M^{j} y$ and $M^{i} y$ numerically, in both cases we will probably end up with a problem similar to the one origin of this section: how to calculate/bound the value of $\left|\partial^{\beta_{1}+2} M^{j} y(\xi) / \partial x^{\beta_{1}+2}-\partial^{\beta_{1}+2} M^{i} y(\xi) / \partial x^{\beta_{1}+2}\right|$ from the values of that function on the set $\left\{x_{l}\right\}$. Solutions to this can be as follows:

(i) Either note that

$$
\begin{aligned}
& \left|\frac{\partial^{\beta_{1}+2} M^{j} y(\xi)}{\partial x^{\beta_{1}+2}}-\frac{\partial^{\beta_{1}+2} M^{i} y(\xi)}{\partial x^{\beta_{1}+2}}\right| \\
& \quad \leq 2 \max \left\{\left|\frac{\partial^{\beta_{1}+2} M^{j} y(\xi)}{\partial x^{\beta_{1}+2}}\right|,\left|\frac{\partial^{\beta_{1}+2} M^{i} y(\xi)}{\partial x^{\beta_{1}+2}}\right|\right\},
\end{aligned}
$$

and, if needed, use the monotonicity properties of all derivatives of $M^{j} y(x)$ and $M^{i} y(x)$ up to $\beta_{1}-1(M$ maps the cone $P$ of $[2,(55)]$ into itself and this implies that all the derivatives of $M f$ up to $\beta_{1}$ have the same sign as $(-1)^{n-k}$; see [2, Proof of Theorem 8]). Such monotonicity properties ensure that the maxima of the absolute value of these derivatives over $\left[x_{l}, x_{l+1}\right]$ are precisely at the extremes $x_{l}$ or $x_{l+1}$.

(ii) Or apply the same principles of (57)-(61), decompose $\partial^{\beta_{1}+2} M^{j} y(\xi) / \partial x^{\beta_{1}+2}-\partial^{\beta_{1}+2} M^{i} y(\xi) / \partial x^{\beta_{1}+2}$ as the sum of the corresponding linear spline and another interpolation error, and obtain an upper bound of $\left|\partial^{\beta_{1}+2} M^{j} y(\xi) / \partial x^{\beta_{1}+2}-\partial^{\beta_{1}+2} M^{i} y(\xi) / \partial x^{\beta_{1}+2}\right|$ from the values of that function at $\left\{x_{l}\right\}$ and a bound for the mentioned interpolation error, which can be calculated using (61) and (65). This gives

$$
\begin{aligned}
& \left|\frac{\partial^{\beta_{1}+2} M^{j} y(\xi)}{\partial x^{\beta_{1}+2}}-\frac{\partial^{\beta_{1}+2} M^{i} y(\xi)}{\partial x^{\beta_{1}+2}}\right| \\
& \quad \leq \max \left\{\left|\frac{\partial^{\beta_{1}+2} M^{j} y\left(x_{l}\right)}{\partial x^{\beta_{1}+2}}-\frac{\partial^{\beta_{1}+2} M^{i} y\left(x_{l}\right)}{\partial x^{\beta_{1}+2}}\right|,\right. \\
& \left.\quad\left|\frac{\partial^{\beta_{1}+2} M^{j} y\left(x_{l+1}\right)}{\partial x^{\beta_{1}+2}}-\frac{\partial^{\beta_{1}+2} M^{i} y\left(x_{l+1}\right)}{\partial x^{\beta_{1}+2}}\right|\right\} \\
& +\frac{\left(x_{l+1}-x_{l}\right)^{2}}{4} \\
& \quad \max \left\{\max \left(\left|\frac{\partial^{\beta_{1}+4} M^{j} y(t)}{\partial x^{\beta_{1}+4}}\right|,\left|\frac{\partial^{\beta_{1}+4} M^{i} y(t)}{\partial x^{\beta_{1}+4}}\right|\right), t\right. \\
& \left.\quad \in\left[x_{l}, x_{l+1}\right]\right\}, \\
& \quad \text { for } \xi \in\left[x_{l}, x_{l+1}\right] .
\end{aligned}
$$

As before, the calculation of the maximum of the last term of (66) can be done from (63)-(64) and using the monotonicity properties of the derivatives of $M^{j} y$ and $M^{i} y$.

Remark 9. Both in the first subinterval $\left[a, x_{1}\right]$ and the last subinterval $\left[x_{m}, b\right]$ of $[a, b]$ the application of Theorem 8 is not possible since

$$
\begin{aligned}
\frac{\partial^{\beta_{1}} M^{j} y(a)}{\partial x^{\beta_{1}}} & =\frac{\partial^{\beta_{1}} M^{i} y(a)}{\partial x^{\beta_{1}}}=\frac{\partial^{\beta_{1}} M^{j} y(b)}{\partial x^{\beta_{1}}} \\
& =\frac{\partial^{\beta_{1}} M^{i} y(b)}{\partial x^{\beta_{1}}}=0,
\end{aligned}
$$

given that $\beta_{1} \leq k-1$. In these subintervals we will need to do the comparison using directly (60).

Remark 10. The estimation of the security margin using Theorem 8, (63)-(64) and either (65) or (66) can be a cumbersome exercise. However, as the examples will show, the bounds that they provide for the extremes $a$ and $b$ for which (1)-(6) has a solution are usually much better than those obtained with the method of Theorem 7 for the same number of iterations.

Remark 11. The arguments of this section are also valid when applied to the functions $M^{j} y$ and $M^{i} y$ and the cone $P$ of (18) for $j>i \geq 0$ (the method of Section 2 assumes that $j>i=0$ ), just replacing $\beta_{1}$ th derivatives by $\mu$ th derivatives. This implies that we can make use of the same functions $y \in P$ to apply both methods and compare results.

\section{Some Examples}

In this section we will present a couple of examples where the results of the Sections 2 and 3 will be used to provide 
progressively better upper and lower bounds of the extremes that make (1)-(6) has a nontrivial solution, for the cases $n=3$ and 4 and different boundary conditions. In all of them the extreme $a$ will be fixed to zero (so as to focus the assessment on the extreme $b$ ) and the integral calculations will be done numerically using a mesh $\left\{x_{l}\right\}$ and applying the trapezoidal rule in each of the subintervals $\left[x_{l}, x_{l+1}\right]$ of the mesh. This also includes the calculation of the derivatives $\left(M^{k} v\right)^{(m)}(x)$, $0 \leq m \leq \mu$, as these can be written as

$$
\begin{aligned}
& \left(M^{k} v\right)^{(m)}(x) \\
& \quad=\int_{a}^{b} \frac{\partial^{m} G(a, b, x, t)}{\partial x^{m}} \sum_{j=0}^{\mu} c_{j}(t)\left(M^{k-1} v(t)\right)^{(j)} d t .
\end{aligned}
$$

The maximum number of iterations has been set to 6 in all examples and up to 3 decimal figures have been provided for each bound.
Example 1. Let us consider the following boundary value problem:

$$
\begin{aligned}
y^{\prime \prime \prime}+y^{\prime}+x y & =0, \quad x \in[0, b] \\
y(0) & =y^{\prime}(0)=y^{\prime}(b)=0 .
\end{aligned}
$$

A possible representation of (69) in a manner that yields an equation $L y=0$ right disfocal in the interval of interest is

$$
\begin{gathered}
y^{\prime \prime \prime}+y^{\prime}=(-1)^{n-k} x y, \quad x \in[0, b] ; \\
y(0)=y^{\prime}(0)=y^{\prime}(b)=0,
\end{gathered}
$$

with $n=3, k=2, \beta_{1}=1$, and $\mu=0$. Equation (70) gives the BVP

$$
\begin{aligned}
y^{\prime \prime \prime}+y^{\prime} & =0, \quad x \in[0, b] ; \\
y(0) & =y^{\prime}(0)=y^{\prime}(b)=0,
\end{aligned}
$$

whose Green function can be calculated following Coppel [23] as

$$
G(x, t)= \begin{cases}\left(\frac{\cos b}{\sin b} \sin t-\cos t\right)(1-\cos x), & 0 \leq x \leq t \leq b \\ (1-\cos t)+\sin t\left(\frac{\cos b}{\sin b}(1-\cos x)-\sin x\right), & 0 \leq t<x \leq b\end{cases}
$$

which coincides with $\partial G^{\mu}(x, t) / \partial x^{\mu}$.

The application of Theorem 3 to the functions $w(x)$ defined in (37) and (39) (considering two different cases $z=$ $b / 2$ and $z=b / 3$ to test the effect of the choice of $z$ in the result of the calculations) gives Table 1 .

Table 1 shows that, as expected, the bounds get improved when the number of iterations grows. Additionally the method provides better upper bounds for $b$ if we pick $z=b / 2$ instead of $z=b / 3$ in the function of (39), for all values of $j$.

On the other hand, the application of the security margin calculated in Theorem 8, using also (66), to determine if the function $M^{6} w-M^{5} w$ belongs to the cone $P$ of (18), gives a value of $b$ between 2.166 and 2.167, much is more precise than the bounds shown in the Table 1 for $j=5,6$. And this occurs regardless of $w(x)$ being that of (37), that of (39) with $z=b / 2$, or that of (39) with $z=b / 3$. This implies that the use of the mentioned security margin to evaluate whether $M^{j} y-M^{j-1} y$ belong to the cone yields better bounds at the expense of complicating the calculations.

Example 2. Let us consider the following boundary value problem:

$$
\begin{aligned}
y^{(i v)}+x y^{\prime}+2 y & =0, \quad x \in[0, b] \\
y(0) & =y^{\prime}(0)=y^{\prime \prime}(0)=y^{\prime \prime}(b)=0 .
\end{aligned}
$$

We can rewrite the problem (73) as

$$
\begin{aligned}
& \begin{array}{l}
y^{(i v)}=-x y^{\prime}-2 y=(-1)^{n-k} x y^{\prime}+(-1)^{n-k} 2 y, \\
x \in[0, b] ; \\
y(0)=y^{\prime}(0)=y^{\prime \prime}(0)=y^{\prime \prime}(b)=0,
\end{array}
\end{aligned}
$$

with $n=4, k=3, \beta_{1}=2$, and $\mu=1$, which, given that $y^{(i v)}=0$ is always right disfocal regardless of the interval $[a, b]$, satisfies all the conditions for the application of the Theorem 3. To do that we first need to determine the Green function of the problem:

$$
\begin{aligned}
& y^{(i v)}=0, \quad x \in[0, b] \\
& y(0)=y^{\prime}(0)=y^{\prime \prime}(0)=y^{\prime \prime}(b)=0 .
\end{aligned}
$$

Following Coppel [23] as we did in Example 1, the mentioned Green function can be calculated as

$$
\begin{aligned}
& G(x, t) \\
& \quad= \begin{cases}-\left(1-\frac{t}{b}\right) \frac{x^{3}}{6}, & 0 \leq x \leq t \leq b, \\
-\frac{t^{3}}{6}+\frac{t^{2}}{2} x-\frac{t}{2} x^{2}+\frac{t}{b} \frac{x^{3}}{6}, & 0 \leq t<x \leq b,\end{cases}
\end{aligned}
$$


TABLE 1: Comparison of bounds for $b$ in Example 1.

\begin{tabular}{lccc}
\hline $\begin{array}{l}\text { Recursivity } \\
\text { index }\end{array}$ & $\begin{array}{c}\text { Bound with } \\
(37)\end{array}$ & $\begin{array}{c}\text { Bound with (39) } \\
\text { and } z=b / 2\end{array}$ & $\begin{array}{c}\text { Bound with (39) } \\
\text { and } z=b / 3\end{array}$ \\
\hline$j=1$ & $b>1.959$ & $b<2.409$ & $b<2.545$ \\
$j=2$ & $b>2.055$ & $b<2.289$ & $b<2.367$ \\
$j=3$ & $b>2.092$ & $b<2.249$ & $b<2.302$ \\
$j=4$ & $b>2.11$ & $b<2.228$ & $b<2.269$ \\
$j=5$ & $b>2.121$ & $b<2.216$ & $b<2.248$ \\
$j=6$ & $b>2.129$ & $b<2.208$ & $b<2.235$ \\
\hline
\end{tabular}

TABle 2: Comparison of bounds for $b$ in Example 2.

\begin{tabular}{lccc}
\hline $\begin{array}{l}\text { Recursivity } \\
\text { index }\end{array}$ & $\begin{array}{c}\text { Bound with } \\
(37)\end{array}$ & $\begin{array}{c}\text { Bound with (39) } \\
\text { and } z=b / 2\end{array}$ & $\begin{array}{c}\text { Bound with (39) } \\
\text { and } z=b / 3\end{array}$ \\
\hline$j=1$ & $b>1.681$ & $b<2.829$ & $b<3$ \\
$j=2$ & $b>1.921$ & $b<2.521$ & $b<2.605$ \\
$j=3$ & $b>2.025$ & $b<2.43$ & $b<2.484$ \\
$j=4$ & $b>2.081$ & $b<2.386$ & $b<2.425$ \\
$j=5$ & $b>2.115$ & $b<2.36$ & $b<2.391$ \\
$j=6$ & $b>2.138$ & $b<2.342$ & $b<2.368$ \\
\hline
\end{tabular}

so that

$$
\begin{aligned}
\frac{\partial^{\mu} G(x, t)}{\partial x^{\mu}} & =\frac{\partial G(x, t)}{\partial x} \\
& = \begin{cases}-\left(1-\frac{t}{b}\right) \frac{x^{2}}{2}, & 0 \leq x \leq t \leq b, \\
\frac{t^{2}}{2}-t x+\frac{t}{b} \frac{x^{2}}{2}, & 0 \leq t<x \leq b .\end{cases}
\end{aligned}
$$

The application of Theorem 3 to the functions $w(x)$ defined in (37) and (39) (considering again two different cases $z=b / 2$ and $z=b / 3$ to test the effect of the choice of $z$ in the result of the calculations) gives Table 2 .

As happened in Example 1, the bounds get improved when the number of iterations grows, and the method provides also better upper bounds for $b$ if we pick $z=b / 2$ instead of $z=b / 3$ in the function of (39), for all values of $j$.

On the other hand, the application of the security margin calculated in Theorem 8, using also (66), to determine whether the function $M^{6} w-M^{5} w$ belongs to the cone $P$ (regardless of $w(x)$ being that of (37), that of (39) with $z=$ $b / 2$, or that of (39) with $z=b / 3$ ), gives a value of $b$ between 2.257 and 2.258, again much more precise than the bounds shown in the Table 2 for $j=5$ or 6 . One can deduce the same conclusion as before: the use of the mentioned security margin to evaluate whether $M^{j} y-M^{j-1} y$ belong to the cone yields better bounds at the expense of complicating the calculations.

\section{Discussion}

The results presented in this paper complement the ones described in [2, Theorems 8-10] when addressing the question of the existence of solutions of those boundary value problems of $n$th order of the type

$$
\begin{aligned}
& y^{(n)}+p_{n-1}(x) y^{(n-1)}+\cdots+p_{0}(x) y=0, \\
& y^{(i)}\left(a^{\prime}\right)=0, \\
& i=0,1,2, \ldots, k-1, \\
& y^{\left(\beta_{i}\right)}\left(b^{\prime}\right)=0, \\
& i=1,2, \ldots, n-k, 0 \leq \beta_{1}<\beta_{2}<\cdots<\beta_{n-k}<n-1,
\end{aligned}
$$

which allow a representation in the form (1)-(6). They overcome the issue of the method of [2, Theorems 8-10] as for the need to compare functions $M^{j} y$ on the entire interval $[a, b]$ for different values of $j$, which gets especially complicated when the calculation of such functions is done numerically.

The method of Section 2 (Theorem 3) has the limitation of requiring $\mu<\beta_{1}$, instead of the original $\mu \leq \beta_{1}$ of [2]. This limitation is not present when applying to $[2$, Theorems $8-10]$ the security margin calculated in Section 3. Another advantage of the use of security margin, as the examples show, is a better speed of convergence of the bounds for $a^{\prime}$ and $b^{\prime}$ towards the values of $a^{\prime}$ and $b^{\prime}$ for which (1)-(6) has exactly a nontrivial solution, compared with the corresponding bounds obtained by means of Theorem 3 . One can conjecture the generality of this behaviour when using a step in the partition $\left\{x_{l}\right\}$ (i.e., the maximum distance between consecutive knots $x_{l}$ and $x_{l+1}$ ) small enough, due to the dependency of the interpolation error with the square of that parameter (see (62)). In any case, the main drawback of the use of the security margin is the difficulty to determine it in each interval $\left[x_{l}, x_{l+1}\right]$, due to the tedious calculations it requires, especially when applying formula (66).

To end up this section, it is worth remarking that in the application of Theorem 3 there is freedom in the choice of the discontinuity point $z$, which can lead to wonder what is the best choice for such a point (i.e., the choice that provides bounds for $a$ and $b$ that converge faster). The two examples presented in this paper show better bounds for the case $z=$ $b / 2$ than for $z=b / 3$, but it is perhaps premature to deduce from that a general principle since this could depend on the concrete problem under assessment, and especially on the value of $k$, which determines the number of derivatives of the solution at the extreme $a$ which are zero (i.e., how "flat" the solution is in the vicinity of $a$ ). More work is therefore required in this area.

\section{Competing Interests}

The authors declare that they have no competing interests. 


\section{Acknowledgments}

This work has been supported by the Spanish Ministerio de Economia y Competitividad Grant MTM2013-41765-P.

\section{References}

[1] M. Greguš, “Third order linear differential equations," in Mathematics and Its Applications, vol. 22, Springer, Amsterdam, The Netherlands, 1st edition, 1987.

[2] P. Almenar and L. Jódar, "Solvability of Nth order linear boundary value problems," International Journal of Differential Equations, vol. 2015, Article ID 230405, 19 pages, 2015.

[3] M. S. Keener and C. C. Travis, "Positive cones and focal points for a class of $n$th order differential equations," Transactions of the American Mathematical Society, vol. 237, pp. 331-351, 1978.

[4] M. G. Krein and M. A. Rutman, "Linear operators leaving a cone invariant in a Banach space," in American Mathematical Society Translation Series, vol. 10, pp. 199-325, American Mathematical Society, Providence, RI, USA, 1962.

[5] M. A. Krasnosel'skii, Positive Solutions of Operator Equations, Fizmatgiz, Moscow, Russia, 1962, English Translation by P. Noordhoff, Groningen, The Netherlands, 1964.

[6] R. D. Gentry and C. C. Travis, "Comparison of eigenvalues associated with linear differential equations of arbitrary order," Transactions of the American Mathematical Society, vol. 223, pp. 167-179, 1976.

[7] K. Schmitt and H. L. Smith, "Positive solutions and conjugate points for systems of differential equations," Nonlinear Analysis: Theory, Methods \& Applications, vol. 2, no. 1, pp. 93-105, 1978.

[8] E. C. Tomastik, "Comparison theorems for second order nonselfadjoint differential systems," SIAM Journal on Mathematical Analysis, vol. 14, no. 1, pp. 60-65, 1983.

[9] D. Hankerson and J. Henderson, "Positive solutions and extremal points for differential equations," Applicable Analysis, vol. 39, no. 2-3, pp. 193-207, 1990.

[10] P. W. Eloe, D. Hankerson, and J. Henderson, "Positive solutions and conjugate points for multipoint boundary value problems," Journal of Differential Equations, vol. 95, no. 1, pp. 20-32, 1992.

[11] P. W. Eloe and J. Henderson, "Focal points and comparison theorems for a class of two-point boundary value problems," Journal of Differential Equations, vol. 103, no. 2, pp. 375-386, 1993.

[12] P. W. Eloe and J. Henderson, "Focal point characterizations and comparisons for right focal differential operators," Journal of Mathematical Analysis and Applications, vol. 181, no. 1, pp. 2234, 1994.

[13] P. W. Eloe, J. Henderson, and H. B. Thompson, "Extremal points for impulsive Lidstone boundary value problems," Mathematical and Computer Modelling, vol. 32, no. 5-6, pp. 687-698, 2000.

[14] P. W. Eloe and B. Ahmad, "Positive solutions of a nonlinear nth order boundary value problem with nonlocal conditions," Applied Mathematics Letters, vol. 18, no. 5, pp. 521-527, 2005.

[15] J. R. Graef and B. Yang, "Positive solutions to a multi-point higher order boundary value problem," Journal of Mathematical Analysis and Applications, vol. 316, no. 2, pp. 409-421, 2006.

[16] J. R. Graef, L. Kong, and H. Wang, "Existence, multiplicity, and dependence on a parameter for a periodic boundary value problem," Journal of Differential Equations, vol. 245, no. 5, pp. 1185-1197, 2008.
[17] X. Zhang, M. Feng, and W. Ge, "Existence and nonexistence of positive solutions for a class of $n$ th-order three-point boundary value problems in Banach spaces," Nonlinear Analysis: Theory, Methods \& Applications, vol. 70, no. 2, pp. 584-597, 2009.

[18] P. Zhang, "Iterative solutions of singular boundary value problems of third-order differential equation," Boundary Value Problems, vol. 2011, Article ID 483057, 2011.

[19] Y. Sun, Q. Sun, and X. Zhang, "Existence and nonexistence of positive solutions for a higher-order three-point boundary value problem," Abstract and Applied Analysis, vol. 2014, Article ID 513051, 7 pages, 2014.

[20] X. Hao, L. Liu, and Y. Wu, "Iterative solution to singular $n$ thorder nonlocal boundary value problems," Boundary Value Problems, vol. 2015, no. 1, article 125, 10 pages, 2015.

[21] P. W. Eloe and J. Ridenhour, "Sign properties of Green's functions for a family of two-point boundary value problems," Proceedings of the American Mathematical Society, vol. 120, no. 2, pp. 443-452, 1994.

[22] G. Hämmerlin and K.-H. Hoffmann, Numerical Mathematics, Springer, New York, NY, USA, 1991.

[23] W. A. Coppel, Disconjugacy, vol. 220 of Lecture Notes in Mathematics, Springer, New York, NY, USA, 1st edition, 1971. 


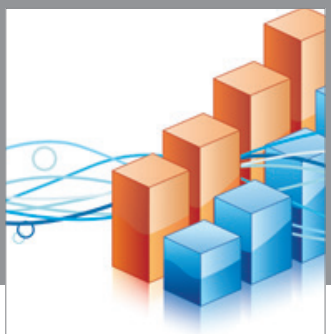

Advances in

Operations Research

vatem alat4

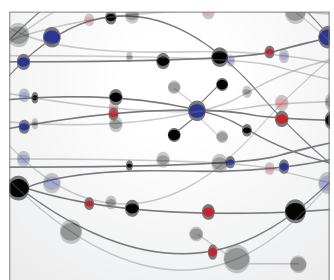

\section{The Scientific} World Journal
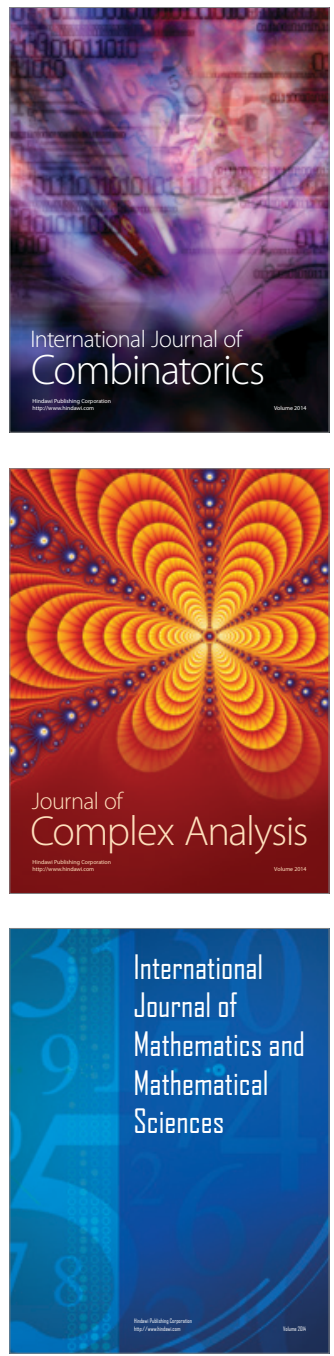
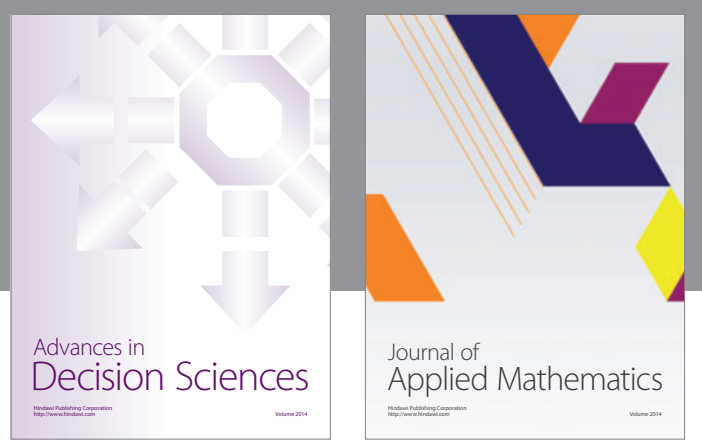

Algebra

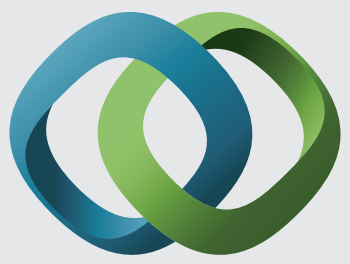

\section{Hindawi}

Submit your manuscripts at

http://www.hindawi.com
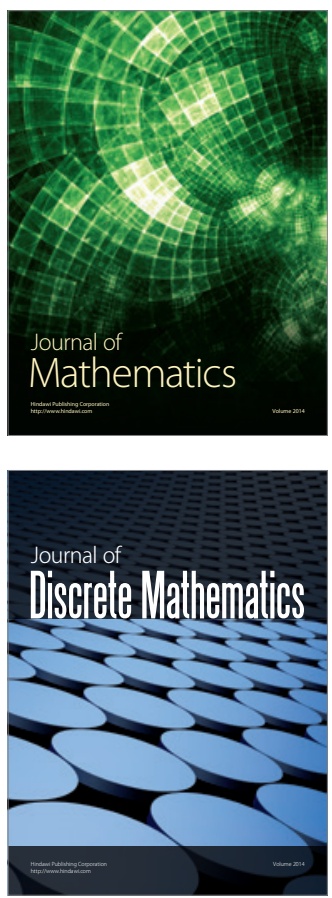

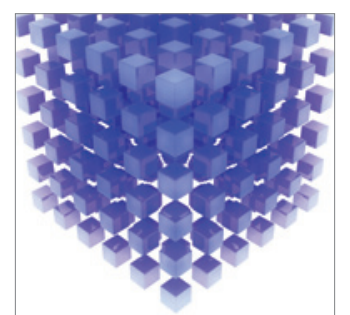

Mathematical Problems in Engineering
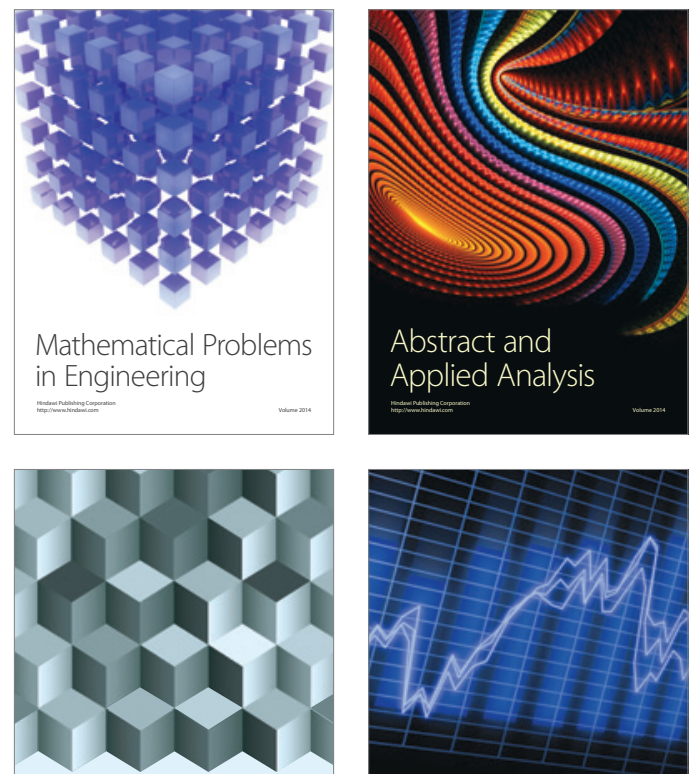

Journal of

Function Spaces

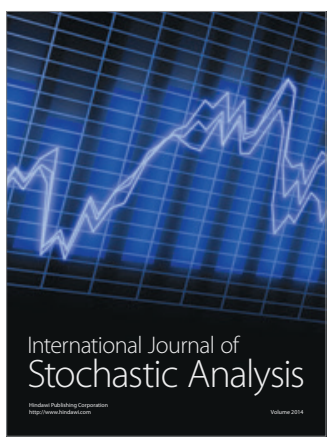

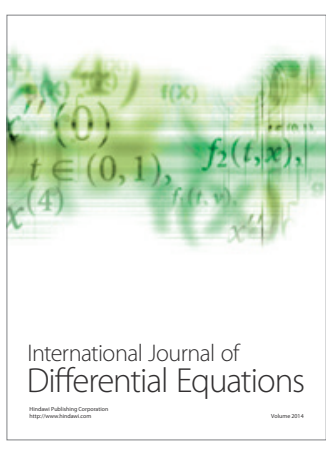
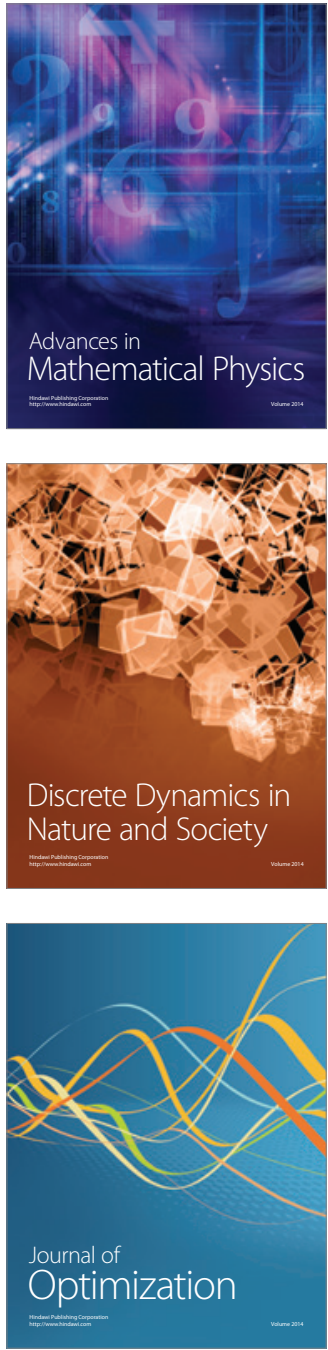